\title{
Complicaciones de la cirugía ginecológica
}

\section{Complications of gynaecological surgery}

\author{
E. Recari, L.C. Oroz, J.A. Lara
}

\section{RESUMEN}

La cirugía ginecólógica está en continuo cambio y por ende algunas de sus complicaciones. La incorporación de la endoscopia ha añadido una nueva dimensión, estando también en este tipo de procedimientos la tasa de complicaciones en relación a la complejidad del mismo. La endoscopia ha representado un gran avance en cirugía y en particular en ginecología, que se beneficia de dos vías de abordaje del aparato genital, la laparoscópica y la histeroscópica.

Estas técnicas tienen unas posibles complicaciones que no son superponibles a la cirugía abierta sino que son específicas de las mismas: creación de neumoperitoneo, colocación de trócares, coagulación a distancia, paso vascular de la glicina empleada en la distensión uterina durante la histeroscopia, etc.

Cuando una paciente acude a urgencias en el postoperatorio o simplemente sufre una complicación en el curso de las 24 ó 48 horas que permanece ingresada, el médico que la atiende debe conocer esta vía de abordaje y sus posibles complicaciones.

Palabras clave. Cirugía ginecológica. Complicaciones. Laparoscopia. Histeroscopia. Abordaje mínimamente invasivo.

\begin{abstract}
Gynaecological surgery is undergoing a process of constant change and therefore some of its complications as well. The incorporation of endoscopy has added a new dimension, with the rate of complications related to its complexity. Endoscopy has meant a great advance in surgery, and specifically in gynaecology, which benefits from two ways of approaching the genital apparatus: laparoscopy and histeroscopy.

These techniques involve risks that are specific to these techniques: the induction of pneumoperitoneum, insertion of trocar, remote coagulation, vascular passage of the glycine employed in uterine relaxation during hysteroscopy, etc....

When a patient comes to Accidents and Emergencies in the postoperative phase, or simply suffers a complication during the 24 or 48 hours that they are hospitalised, the doctor who attends her must be acquainted with this course of approach and its possible complications.
\end{abstract}

Key words. Gynaecological surgeries. Complications Laparoscopy. Histeroscopy. Minimally invasive approach.
Servicio de Obstetricia y Ginecología. Hospital Virgen del Camino. Pamplona.

\section{Correspondencia:}

Eva Recari Elizalde

Avda. Eulza, 73-1ํA

31010 Barañáin (Navarra)

Tfno. 948703919-619242957

E-mail: erecari@hotmail.com 


\section{INTRODUCCIÓN}

La tasa de mortalidad operatoria en las intervenciones ginecológicas es baja, inferior al 1\%. Un estudio retrospectivo de 1,45 millones de pacientes halló una mortalidad del $0,2 \%$ en los primeros 30 días de ingreso ( $5,1 \%$ en pacientes oncológicos versus $0,1 \%$ en pacientes sin cáncer) ${ }^{1}$.

La tasa de complicaciones asociadas a la cirugía varía entre el $0,2 y$ el $26 \%{ }^{2}$. Las complicaciones más frecuentes de la cirugía ginecológica están relacionadas con el daño visceral (vejiga, recto, uréteres) y de los grandes vasos pélvicos. Son más frecuentes en la cirugía oncológica o cuando la anatomía está distorsionada debido a infección o a endometriosis. El factor más importante que determina el éxito de su tratamiento, es el reconocimiento precoz de las complicaciones. Por ello, es tan importante la observación sistemática y cuidadosa del post-operatorio, particularmente: pulso, presión sanguínea, función respiratoria, temperatura, diuresis, hemograma, etc.

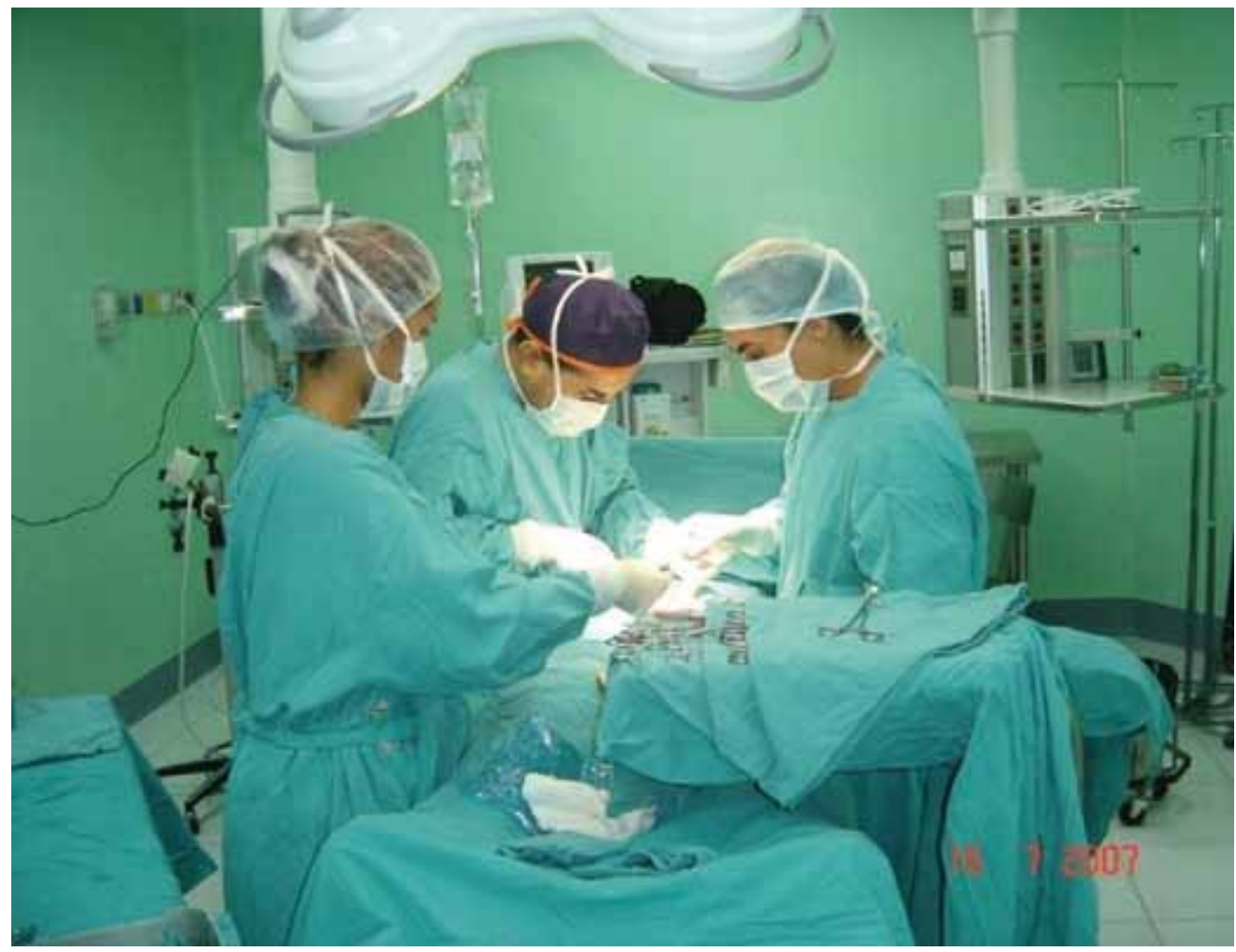

Figura 1. Cirugía abierta.

Harris ${ }^{3}$ publicó la evolución histórica de las complicaciones asociadas a la histerectomía describiendo una disminución en la tasa de trasfusiones, la de infecciones del tracto urinario y un incremento en la tasa de lesiones vesicales. Asimismo, ha identificado cuatro factores asociados con una mayor tasa de complicaciones: edad, enfermedad médica preexistente, obesidad y cáncer. Se realiza la descripción de las complicaciones en la cirugía ginecológica generales (abierta), haciendo paréntesis en aquellas complicaciones propias de la vía de acceso (endoscópica y vaginal) (Figs. 1-3). 


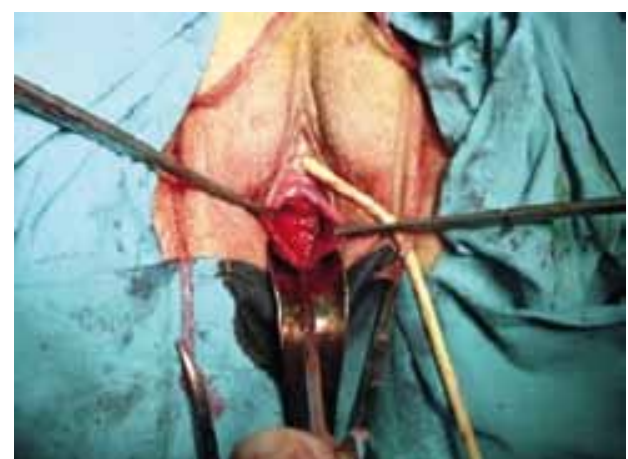

Figura 2. Cirugía vaginal.

\section{COMPLICACIONES DE LA CIRUGÍA GINECOLÓGICA ABIERTA}

\section{Hemorragia post-quirúrgica}

La hemorragia post-operatoria puede manifestarse como una pérdida sanguínea por los drenajes o por hipovolemia manifestada con mareos-hipotensión o en control analítico, a las 4 -6 horas post intervención.

\section{Hemorragia precoz}

Puede producirse durante las primeras 24 horas (habitualmente durante las primeras 8-10 horas) en la pared abdominal, la cavidad abdominal, vagina, tejidos blandos de la pelvis, vejiga o de varios sitios al mismo tiempo. Se debe sospechar un hematoma de la pared abdominal si existe un dolor local, intenso, que puede deformar la pared. En hematomas pequeños es habitual una actitud expectante. Los hematomas grandes, sobre todo si son progresivos, requieren intervención inmediata con ligadura vascular si es posible, limpieza y drenaje.

\section{- Hemorragia vaginal}

Es más frecuente después de la histerectomía vaginal que de la abdominal. Suele comenzar varias horas después de la intervención y proceder de la rama cervical o vaginal de la arteria uterina, de los ángulos vaginales de la incisión o de las incisiones de la colporrafia. El taponamiento suele ser útil si el sangrado es leve pero si el sangra-

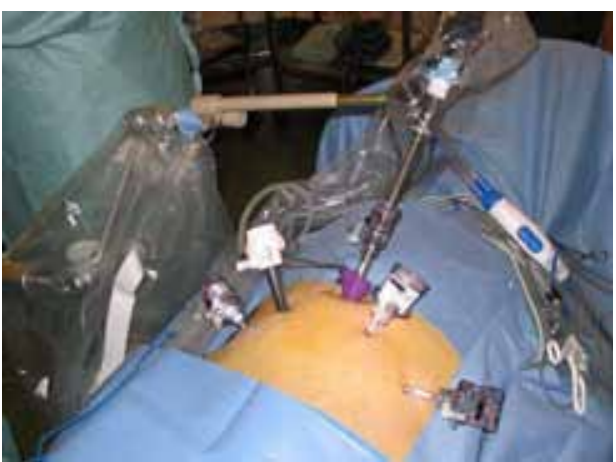

Figura 3. Cirugía laparoscópica.

do es arterial es necesaria una exploración bajo anestesia.

\section{- Hemorragia extraperitoneal}

La hemorragia en el tejido conjuntivo pélvico es difícil de tratar. Se caracteriza por una masa tensa y elástica en la pelvis, tumefacción a nivel del ligamento inguinal y una matidez sobre todo en los flancos. Afortunadamente suelen ceder espontáneamente. El sangrado suele ser venoso y su origen difícil de localizar. Generalmente es suficiente con la evacuación del hematoma y eliminación del tejido necrótico.

\section{- Hemorragia vesical}

Suele producirse en intervenciones urológicas, por la colocación de un drenaje supra púbico o la lesión del órgano durante la cirugía. Se suele controlar con medidas conservadoras (sonda vesical, y lavados vesicales continuos). Si no fuese suficiente se debe tratar con una cistotomía suprapúbica.

\section{Hemorragias tardías}

Ocurren habitualmente entre el $7^{\circ}$ y $21^{\circ}$ días; habitualmente son hemorragias del muñón vaginal después de una histerectomía. Las causas más frecuentes son: reabsorción de los hilos de sutura, necrosis de los tejidos locales, tromboflebitis séptica, tratamiento anticoagulante y ocasionalmente coito antes de la cicatrización. Es recomendable la exploración bajo anestesia para localizar el punto sangrante. La 
intervención puede ser dificultosa por la friabilidad y debilidad de los tejidos. En casos excepcionales es necesario realizar una laparotomía y ligar la arteria uterina en su origen.

\section{Complicaciones infecciosas}

Las infecciones postoperatorias, aunque han disminuido en su gravedad siguen siendo frecuentes, graves para el paciente y onerosas para el sistema. Hay que tener en cuenta que las operaciones con apertura de la vagina están asociadas a la contaminación con las bacterias residentes en aquella. Muchas de las infecciones postoperatorias son polimicrobianas y están causadas por microbios residentes en la vagina, así como por gérmenes anaerobios.

La profilaxis antibiótica ha sido ampliamente adoptada en ginecología, sobre todo después de que diferentes estudios prospectivos en la década de los 80 han demostrado su efectividad para reducir la tasa de infecciones postoperatorias. Una compilación de 16 de estos estudios demostró que la tasa de infecciones tras histerectomía vaginal pasó del 32 al $6 \%$ cuando se administraba profilaxis ${ }^{4}$ y otro meta-análisis de 25 estudios demostró que dicha tasa pasaba del 21 al $9 \%$ en casos de histerectomía abdominal (Evidencia I) ${ }^{5}$.

Los agentes usados con más frecuencia en ginecología para la profilaxis antibiótica son las cefalosporinas. Son efectivas, bactericidas, no tóxicas y baratas. Ningún estudio ha demostrado que antibióticos más recientes (y más caros), sean más efectivos ${ }^{4}$. Es suficiente una dosis administrada durante las dos horas previas a la incisión, sin que haya evidencia (excepto para aquellas intervenciones que se alargan en el tiempo) de mayor eficacia de dosis repetidas.

\section{Infecciones post-operatorias precoces}

El primer signo de infección suele ser la fiebre precoz elevada. La fiebre que aparece en las primeras 24-48 horas después de la intervención, habitualmente no se debe a infección, puede tener un origen inespecífico y no ser determinado con certeza y, por lo tanto, el tratamiento antibiótico no debe instaurarse solamente por la fiebre ${ }^{6}$. Otras causas de fiebre incluyen la atelecta- sia, la neumonía por aspiración, la infusión de líquidos contaminados, las lesiones ureterales o intestinales o infecciones preexistentes del útero, pelvis o tracto urinario. La infección de los tejidos quirúrgicos por el estreptococo hemolítico y más raramente fascitis necrotizante.

\section{Infecciones post-operatorias tardías}

Aparecen varios días después de la intervención siendo los signos de localización más evidentes. Los procesos que se pueden presentar son: infección de la herida quirúrgica, infecciones pélvicas, abscesos pélvicos, tromboflebitis pélvica séptica, infecciones de los catéteres, infecciones del tracto urinario, bronconeumonía y fiebre medicamentosa.

\section{Infecciones pélvicas}

Los abscesos pélvicos suelen se secundarios a celulitis de la cúpula vaginal .Los síntomas suelen presentarse a los 5-10 días después de la cirugía y consisten, en fiebre y dolor abdominopélvico. Suele existir leucocitosis con desviación izquierda y el tacto vaginal provoca dolor a la paciente; puede existir secreción purulenta a través de los bordes de la herida quirúrgica. La ecografía o el TAC suele confirmar o descartar la presencia de un abceso. El tratamiento consiste en antibióticoterapia (ampicilina asociada a un aminoglucósido-en general la gentamicina, añadiendo anaerobicidasclíndamicina o metronidazol, en función de los hallazgos en cada paciente) y drenaje de la colección purulenta (vía vaginal o percutánea, en función de su localización).

\section{Infecciones urinarias}

$\mathrm{Su}$ frecuencia puede alcanzar el $40 \%$ sin profilaxis antibiótica ${ }^{7}$. La mayoría son leves, siendo rara la pielonefritis. El tratamiento consiste en la antibióticoterapia adecuada.

\section{Infecciones respiratorias}

La neumonía post-operatoria no es frecuente, debido a que la mayoría de pacientes suelen tener una buena condición 
preoperatoria y al frecuente uso de la anestesia locorregional. Se consideran factores de riesgo la intubación orotraqueal y anestesia general, obesidad, edad avanzada, enfermedades previas y uso de tabaco. Su prevención incluye la movilización precoz, la estimulación de la paciente a toser y a realizar respiraciones profundas.

\section{Flebitis}

La incidencia de infecciones secundarias a catéteres endovenosos puede ser hasta del $30 \%$. Su prevención incluye su colocación estéril, bajo indicación estricta, reemplazo cada tres días y retirada precoz. El tratamiento es sintomático. Cuando existe evidencia de infección sistémica es útil el uso de antibióticos con actividad antiestafilococo.

\section{Sepsis}

La sepsis es una respuesta sistémica a la infección. Se considera severa cuando existe algún signo de disfunción orgánica (como acidosis metabólica, encefalopatía, oliguria, hipoxemia, hipotensión, o alteraciones de la coagulación). Cuando la respuesta sistémica es muy importante puede producirse un shock o incluso, cuando falla la perfusión orgánica, se produce el llamado síndrome de sepsis o síndrome orgánico múltiple. La sepsis está producida por los mismos gérmenes que producen las infecciones descritas antes, por lo que suele tratarse de infecciones polimicrobianas en las cuales las bacterias y sus toxinas han alcanzado el torrente circulatorio y circulan por él. El tratamiento se inicia con la erradicación precoz y agresiva de la fuente de infección, con antibióticos sistémicos y cirugía si está indicada, así como la puesta en marcha de todas las medidas de soporte orgánico necesarias. Cuando existe evidencia de fallo orgánico es obligado el ingreso en una unidad de vigilancia intensiva.

\section{Complicaciones digestivas}

\section{Íleo paralítico y obstrucción intestinal}

En la mayoría de casos el aparato digestivo recupera su función normal al tercer día del post-operatorio; de hecho se calcula que el intestino delgado recupera su funcionalismo en 6 a 12 horas, el estómago en 12 a 24 horas y el intestino grueso en 48 a 72 horas $^{8}$, aunque la manipulación excesiva del intestino durante la cirugía, el uso de narcóticos y otras circunstancias pueden alargar estos periodos. Sin embargo, el inicio precoz (a las 6 horas de la intervención) de la ingesta hídrica o dieta ligera no se ha asociado con un aumento de la incidencia de íleo paralítico ${ }^{9}$. El íleo implica disminución o ausencia de la función propulsiva intestinal debido a parálasis u obstrucción mecánica. Si persiste, el intestino comienza a distenderse y su contenido queda secuestrado con gran pérdida de agua, electrolitos y albúmina ocasionando hipovolemia, elevación del diafragmática, insuficiencia respiratoria y shock. Se produce en el $2 \%$ de todas las histerectomías abdominales por patología benigna y sólo en el $0,2 \%$ de las vaginales, aunque puede llegar al $4 \%$ cuando se tienen en cuenta todas las cirugías ${ }^{10}$. La obstrucción intestinal tiene una incidencia del $0,1-1 \%$ y puede aumentar hasta el $20 \%$ cuando se añaden factores de riesgo como cirugía oncológica o radioterapia postoperatoria ${ }^{11}$. Las adherencias son la causa más frecuente de la obstrucción intestinal, tanto cuando ésta se localiza a nivel del intestino grueso $(30 \%)$ como cuando se localiza a nivel del intestino delgado (60\%). Existen datos que sugieren que la histerectomía es una de las operaciones previas más frecuentes en estas pacientes. El pronóstico depende de la duración del íleo. Es importante el diagnóstico y tratamiento precoces. Se distingue durante el periodo postoperatorio entre el íleo temprano y el tardío. Dependiendo de la causa y de los síntomas en: a) íleo mecánico (obstrucción intestinal); b) íleo paralítico, funcional o dinámico y c) íleo mixto.

\section{- Diagnóstico}

Los síntomas de un íleo funcional son: dolor abdominal, sensación de plenitud, distensión de la porción superior del abdomen, vómitos y náuseas. No hay ruido intestinal y la percusión de la pared demuestra la existencia de líquido. En la radiografía de abdomen puede observarse dilatación de asas de intestino delgado con ausencia de gas en las asas posteriores a la obstrucción (en caso de que exista). Asimismo, en ca- 
sos de obstrucción, suele existir leucocitosis con desviación izquierda.

La obstrucción por estrangulamiento de un asa producida por adherencias intraperitoneales ocurre entre el $5^{\circ}-7^{\circ}$ día. El cuadro clínico puede asociarse con una obstrucción parcial o completa (íleo mecánico). Los síntomas son dolor cólico abdominal, vómitos y un aumento de los ruidos peristálticos con sonidos metálicos. Si se produce la encarcelación del asa aparecen de forma aguda.

- Tratamiento

- Descompresión del estómago e intestino con una sonda nasogástrica conectada a aspiración.

- Cuando la atonía postquirúrgica es más prolongada es conveniente realizar radiografía de abdomen en vacío ortoestática.

- Corregir cualquier trastorno del equilibrio hidroelectrolítico y mantener un equilibrio ácido-base adecuado.

- Estimulación del peristaltismo con agentes colinérgicos. También se pueden administrar laxantes o enemas.

- Si las medidas conservadoras y la aspiración gastrointestinal no mejoran el cuadro en 48 horas será conveniente realizar nueva cirugía para buscar y solucionar la causa del íleo.

\section{Lesiones intestinales quirúrgicas}

La posibilidad de una lesión quirúrgica del intestino en el curso de una intervención ginecológica siempre está presente, pero el riesgo aumenta en casos de endometriosis, enfermedad inflamatoria pélvica, cáncer, adherencias importantes o pacientes con antecedentes de radioterapia.

Si se identifica la lesión en el acto operatorio, debe procederse a su corrección quirúrgica. Si no se ha identificado, pero tras la intervención aparecen signos de sospecha de lesión intestinal (colecciones líquidas, abscesos, íleo que no responden al tratamiento conservador, etc...), debe plantearse la reintervención urgente si hay algún signo de sepsis o antecedentes de radioterapia. $\mathrm{Si}$ no hay signos de sepsis se puede intentar el tratamiento conservador, con aspiración de las secreciones, colocación de drenajes si es posible y seguimiento cuidadoso.

\section{Dehiscencias y evisceración}

Estos términos implican la separación sintomática o asintomática de los bordes de las incisiones quirúrgicas, con o sin signos de infección. Existen algunos síntomas y signos prodrómicos, especialmente un drenaje seroso o serosanguinolento por la herida. La incidencia es del 0 al 3\%.

Para producirse una dehiscencia es preciso que exista una infección, junto con factores mecánicos asociados a complicaciones abdominales y pulmonares. Los factores son: tipo de incisión y técnica de sutura utilizada; demasiadas suturas excesivamente juntas o con tensión excesiva; aumento de presión intraabdominal (tos, vómito, íleo), las complicaciones de la herida (abscesos sobre todo si hay peritonitis, hematoma o serosa), o la obesidad mórbida. La edad, caquexia, déficit vitamínico, anemia y ciertos medicamentos tienen una implicación más controvertida.

La elección de la incisión y de la técnica de cierre apropiada ayudan a prevenir la dehiscencia y eventración. Las características de las pacientes nos orientarán para tomar medidas convenientes para prevenir la complicación (material de reabsorción lento o no reabsorbible, suturas e descarga, si hay infección se deja piel y grasa abierta...). La identificación y tratamiento precoz es muy importante.

El manejo incluye el tratamiento de los factores contribuyentes tales como la peritonitis y el cierre de la incisión tan rápidamente como sea posible. Si protruye el intestino debe ser inmediatamente cubierto con una compresa estéril y húmeda. Una faja evitará que aumente la dehiscencia hasta que la paciente se lleve de nuevo al quirófano.

\section{Complicaciones tromboembólicas}

Se calcula que hasta el $20 \%$ de las muertes postoperatorias son debidas a embolismo pulmonar.

\section{Trombosis venosa}

Este proceso constituye la causa subyacente de la mayoría de casos de tromboembolismo pulmonar. En cirugía ginecológi- 
ca las venas que con mayor frecuencia lo presentan son las de las piernas y las de la pelvis. El diagnóstico es en ocasiones complicado: menos de la mitad de las pacientes con dicho proceso presentan síntomas, y sólo el $40 \%$ de las pacientes con sospecha clínica ven confirmado su diagnóstico por ecografía Doppler. El tratamiento se debe realizar con heparina, idealmente en infusión continua después de una dosis de carga de 5.000-10.000 unidades. Se debe monitorizar el tiempo de cefalina hasta conseguir mantenerlo entre 1,2 y 2 veces el control. La heparina debe mantenerse durante 5-7 días y posteriormente se pasará a anticoagulantes orales o heparina de bajo peso molecular durante 3 a 6 meses si no existen otros factores de riesgo.

\section{Tromboembolismo pulmonar}

El diagnóstico requiere un alto grado de sospecha dado que tanto los síntomas como los signos no son ni sensibles ni específicos: disnea, dolor pleural, angustia, taquicardia, cianosis, síncope, tos, hemoptisis, fiebre, hipotensión y otros. Las pruebas complementarias básicas incluyen radiografía de tórax, ECG (aparición de una onda S en DI, una onda Q en V3 e inversión de $\mathrm{T}$ en $\mathrm{V} 3$, aunque en general es normal) $\mathrm{y}$ una gasometría (que en casos severos puede mostrar una disminución de la $\mathrm{Pa} \mathrm{O}_{2}$, aumento de la $\mathrm{PaCO}_{2}$ y disminución del $\mathrm{pH}$ ). El diagnóstico definitivo se obtiene con una gammagrafía de ventilación-perfusión o con un angiograma pulmonar con TAC.

El tratamiento requiere ingreso en UCI y descoagulación a dosis plenas, que debe administrarse mediante una pauta similar a la descrita para la trombosis venosa profunda ${ }^{12}$.

\section{Complicaciones urinarias}

Las cifras de lesión vesical y ureteral son más altas durante los procesos oncológicos o en cirugías por procesos benignos altamente específicos.

Se calcula que más del $75 \%$ de las lesiones ureterales son debidas a la cirugía ginecológica, siendo más del $70 \%$ de estas intervenciones histerectomías abdominales. Se calcula que se produce una lesión vesical en algo menos del $2 \%$ de las histerectomías abdominales y en algo menos del $0,5 \%$ de las vaginales. Los procedimientos laparoscópicos parecen tener una cifra más alta de daño ureteral que la cirugía abierta.

\section{Lesión vesical}

Si se sospecha lesión durante la intervención, ésta puede ser localizada a través de inyección intravenosa de índigo carmín, o de forma retrógrada con instalación de azul de metileno a través de una sonda uretral. Daños menores pueden requerir el uso de la cistoscopia. En caso de que se compruebe la lesión durante el mismo acto operatorio es necesaria la reconstrucción inmediata, con una o dos suturas continuas de material reabsorbible y dejar la sonda vesical de cuatro a siete días.

\section{Lesión ureteral}

Para prevenir su lesión es fundamental conocer bien la anatomía y los lugares en que es más frecuente la misma, en general a nivel del ligamento infundíbulo-pélvico, a nivel de los ligamentos útero-sacros y a nivel de la arteria uterina. Tanto la práctica de pielografía endovenosa como la colocación de catéteres ureterales de forma preoperatoria para prevenir estas lesiones no debe hacerse de forma rutinaria, sino en función de las características de cada paciente $^{13}$. La mejor opción ante sospecha es la visualización directa de los uréteres. Si no es posible (vía vaginal, obesidad...) puede realizarse cistoscopia para demostrar la salida de colorante azul a través de los uréteres previa administración endovenosa de $5 \mathrm{ml}$ de índigo carmín. La comprobación de lesión en el acto operatorio requiere su reconstrucción inmediata. Si se sospecha la lesión en el post-operatorio hay que realizar una pielografía intravenosa. En caso de que ésta nos confirme la sospecha, debe resolverse con urgencia solicitando la ayuda de un urólogo experimentado.

\section{Formación de fístulas}

\section{Tracto urinario}

La mayoría de las fístulas ocurren tras histerectomías por procesos benignos, 
dado que estos procedimientos son más frecuentes que la cirugía del cáncer. Sin embargo, el riesgo de fístula es más alto tras la histerectomía radical debido a la propia cirugía, a la presencia del tumor y en algunos casos a los cambios inducidos tras radioterapia.

Las fístulas vesicales o ureterales ocurren en menos del $1 \%$ de las cirugías radicales. La fístula se localiza por cistoscopia, pielografía endovenosa y estudios retrógrados del uréter. En las fístulas grandes la paciente habitualmente refiere pérdida espontánea de orina. En estos casos las fístulas son fácilmente visibles mediante examen con espéculo, pero las pequeñas pueden ser difíciles de detectar. La instilación de carmín índigo o azul de metileno en la vejiga puede ser útil en los casos en que el diagnóstico no está claro y permite distinguir entre fístulas vesicovaginales y ureterovaginales. Se colocan algodones sueltos en toda la longitud de la vagina y se indica a la paciente que camine durante unos 10 ó15 minutos. Si el algodón más externo se tiñe la paciente tiene, con más probabilidad, incontinencia de estrés o de urgencia. Si hay una fístula ureterovaginal, el algodón más interior está húmedo pero no coloreado. La tinción de los algodones superiores sugiere una fístula vesicovaginal ${ }^{14}$. Las fístulas pequeñas no asociadas con radiación o cáncer pueden cicatrizar espontáneamente. Más del 30\% de las fístulas ureterovaginales cierran solas y no requieren cirugía, si no hay signos de obstrucción Las fístulas pequeñas vesicovaginales postquirúrgicas también suelen cerrar espontáneamente con el uso continuo durante 4 a 6 semanas de sonda urinaria.

Si su diagnóstico no es precoz (48-72 horas) se debe demorar la reparación de tres a cuatro meses, hasta que se complete la demarcación del tejido cicatricial.

\section{Tracto gastrointestinal}

Las fístulas gastrointestinales tras la cirugía ginecológica son unas complicaciones raras. Suelen debutar a los 10-14 días de la intervención, con picos febriles sin foco claro y sin respuesta a los antibióticos. El diagnóstico es radiográfico, tras la inyección de contraste en la fístula y la realización de series de intestino delgado y grueso. Si la fístula drena al exterior y en pequeña cantidad, la nutrición parenteral contribuye a la cicatrización. Si ésta no ocurre, se debe realizar laparotomía para su reparación. Las fístulas rectovaginales pueden aparecer tras histerectomías y cirugías de prolapso y suelen localizarse en la mitad superior de la vagina. La mayoría se descubren cuando se separan los labios y se examina la vagina con un espéculo, y a menudo ésta contiene material fecal. Si son pequeñas puede ayudar a su diagnóstico el taponamiento del recto con el balón de un catéter de Foley seguido de la instilación en el recto de azul de metileno, índigo carmín o aire. Se recomienda el estudio radiográfico de la fístula (fistulografía). La reparación de la fístula sólo debe acometerse cuando la reacción inflamatoria alrededor de la lesión se ha solucionado por completo, lo que conlleva un mínimo de 3 meses, pudiendo durar hasta 12 meses.

\section{COMPLICACIONES DE LA CIRUGÍA LAPAROSCÓPICA}

La endoscopia ha representado un gran avance en cirugía y en particular en ginecología, que se beneficia de dos vías de abordaje del aparato genital: la laparoscópica y la histeroscópica (Figs. 4 y 5).

A menudo, la introducción de nuevas técnicas, aun aplicadas sobre conocidos campos quirúrgicos conduce a una proliferación de accidentes y complicaciones. Una revisión incluía sobre 1,5 millones de pacientes intervenidos, complicaciones en el 0,1 al $10 \%$ de los procedimientos ${ }^{15}$. No obstante, la laparoscopia ginecológica ha alcanzado en la actualidad un grado suficiente de madurez y permite realizar más de la mitad de la cirugía ginecológica, incluida la oncológica, ya que a través de estas vías se pueden efectuar con similar precisión las técnicas quirúrgicas con las conocidas ventajas de la vía endoscópica: menor traumatismo quirúrgico, menor hemorragia intraoperatoria, menor analgesia, menor coste y mejor y más rápida recuperación laboral y social. 


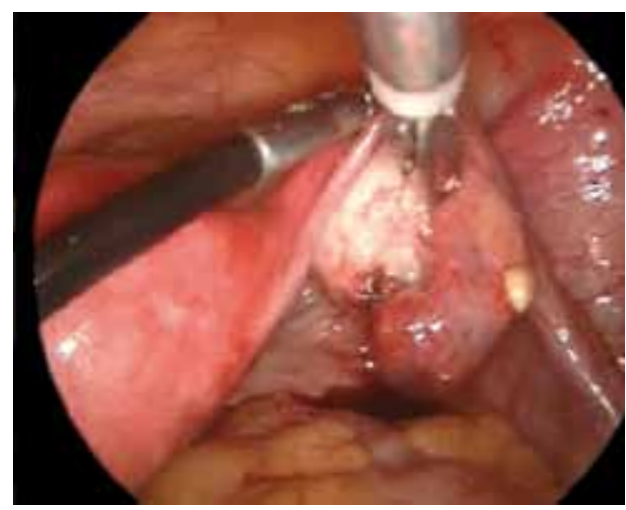

Figura 4. Gestación ovárica.

Estas técnicas tienen unas complicaciones que no son superponibles al la cirugía abierta, sino que son específicas de las mismas: creación de neumoperitoneo, colocación de trócares, coagulación a distancia, paso vascular de glicina empleada en la distensión uterina durante la histeroscopia, etc.

\section{Lesiones durante la insuflación de $\mathrm{CO}_{2}$}

La laparoscopia puede ser abierta o cerrada (convencional). Hay controversias sobre si es más prudente practicar todas las laparoscopias "abiertas", o si es preferible, la técnica convencional en casos de bajo riesgo de adherencias periumbilicales -que son la mayoría de los casos. Actualmente no hay evidencia de menores complicaciones en una técnica que en otra ${ }^{16}$. Una revisión de la literatura desde 1975 al 2002 sobre lesiones producidas por la entrada (aguja de Veress, trócar subumbilical) publicó un 0,3 a 1,3\% de lesiones viscerales y de un 0,07 a $4,7 \%$ o de lesiones vasculares $^{17}$. Los procedimientos en pacientes con cirugia previa o con enfermedad intraabdominal (endometriosis, EPI) están asociados a mayor riesgo de complicaciones. Las condiciones que incrementan el riesgo incluyen: distensión intestinal, grandes masas abdominales, adherencias, enfermedad cardiopulmonar o hernia diafragmática.

Por último, el número de complicaciones está asociado de forma inversamente proporcional a la experiencia del cirujano, dada por el número de intervenciones realizadas.

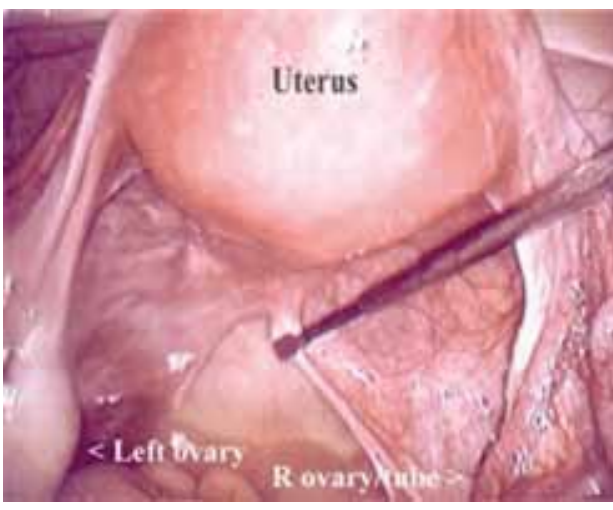

Figura 5. Anatomía pélvica.

\section{Insuflación extraperitoneal de $\mathrm{CO}_{2}$}

Una introducción defectuosa de la aguja de Veress (instrumento clásico para la creación del neumoperitoneo previo a la colocación de los trócares) ya sea, por una penetración insuficiente de la pared abdominal, o una incorrecta inclinación/ elevación de la misma, pueden ocasionar la distensión gaseosa del espacio subcutáneo, preperitoneal o a nivel de epiplon, lo que provoca un enfisema que dificulta la colocación de los trócares y la visión posterior. Es la complicación más frecuente y habitualmente cambiando el punto de inserción de la aguja de Veress se consigue una buena entrada intraperitoneal y el enfisema se resuelve de forma espontánea. No obstante, en algún caso un aumento de presión de $\mathrm{CO}_{2}$ en la sangre de la paciente puede obligar a maniobras de hiperventilación mecánica por parte del anestesista. Para verificar su correcta posición intraabdominal se debe verificar la presión y el flujo de $\mathrm{CO}^{2}$ (la primera será baja y el otro alto). También se puede inyectar suero fisiológico por la aguja y si éste no se recupera, es que probablemente estamos en la cavidad.

\section{Perforación de víscera hueca}

- Tracto gastrointestinal. Las lesiones del tracto gastrointestinal ocurren en 1-2 \% de los procedimientos laparoscópicos.

- Las lesiones gástricas sólo pueden producirse si hay una distensión gástrica, casi siempre por error en la intu- 
bación anestésica. Si por la aerofagia que presenta la paciente, y la hipoxia por la falta de ventilación se detecta la punción inadvertida de la aguja de Veress, no se requiere más tratamiento que colocar una sonda nasogástrica y venoclisis.

- Las lesiones intestinales (delgado y grueso) son poco frecuentes, pero pueden ocurrir incluso en manos expertas (sobre todo en presencia de síndrome adherencial). El test de aspiración por la aguja de Veress puede evidenciar la salida del contenido intestinal, o puede detectarse la salida de gases por el ano. La retirada de la aguja no comporta riesgo de peritonitis, pero requiere irrigación profusa de la zona con aspiración y una simple profilaxis con anaerobicidas. A menudo (40-50\%) la perforación puede pasar inadvertida y mostrarse en el post-operatorio de forma solapada como íleo paralítico, fiebre o incluso estados de sepsis, sin que la clínica de peritonismo abdominal sea aparente (y, consecuentemente, de difícil diagnóstico). Así, en cualquier caso de evolución desfavorable a las 48-72 horas del post-operatorio es necesario sospechar la complicación y recurrir a exploraciones como la ecografía o el TAC para valorar entre otros signos la presencia de líquido intraabdominal libre.

- Lesiones vesicales. La vejiga soporta bien los traumas con agujas. No obstante, previamente a cualquier maniobra resulta preceptivo el sondaje y vaciado de la misma.

\section{Lesiones vasculares}

El daño vascular más frecuente es por laceración de vasos mesentéricos al introducir la aguja de Veress a través del ombligo, particularmente si existen adherencias de epiplon a dicho nivel.

La introducción de la aguja de Veress en un vaso de gran calibre se detecta fácilmente por la salida de sangre a través de la misma, o durante la primera maniobra de comprobación. La insuflación directa de $\mathrm{CO}_{2}$ al torrente sanguíneo puede terminar con una grave y fulminante embolia gaseo- sa. No se han encontrado referencias de la punción inadvertida de la aorta con la aguja de Veress.

\section{Lesiones provocadas por los trócares}

\section{Lesiones vasculares}

La punción de grandes vasos abdominales (aorta, cava, mesentérica inferior) al introducir el primer trócar trans-umbilical no es superior al 0,3-0,9\%. Son más probables en los casos de pacientes muy delgadas o niñas, ya que hay que tener en cuenta, que la posición de los grandes vasos sobre la columna está sólo a un tercio de profundidad del total del grosor externo del tronco. Resulta imprescindible una buena distensión abdominal con el fin de aumentar esta distancia, es decir, un neumoperitoneo con unas presiones mínimas entre 15 y $20 \mathrm{~mm} \mathrm{Hg}$. Debe introducirse el trócar en posición horizontal (en Trendelenburg se acerca la aorta al ombligo) y levantando más la pared abdominal cuanto más delgada sea la paciente. El tratamiento de estas complicaciones exige una laparotomía inmediata, compresión eficaz y ligadura posterior, a ser posible, por un cirujano vascular.

Los trócares accesorios colocados lateralmente pasan cerca de los grandes vasos iliacos, pero al colocarlos con visión intraabdominal el riesgo disminuye. No obstante, las lesiones vasculares más frecuentes son las de los vasos epigástricos inferiores que pueden coincidir con la colocación de los trócares. La transiluminación de la pared abdominal, salvo en grandes obesas, permite evitar los vasos epigástricos tanto superficiales como inferiores, pero para ello hay que oscurecer el quirófano y aplicar la óptica iluminada en la zona a incidir. Los daños vasculares pueden no ser apreciados de forma inmediata debido a que el sangrado es con mayor frecuencia retroperitoneal.

\section{Lesiones intestinales}

Es una de las complicaciones más serias, porque puede no ser diagnosticada en el quirófano lo que incrementa el riesgo de peritonitis fecal y muerte. 


\section{- Lesiones de intestino grueso}

Complicación rara $(0,1 \%)$ y si se produce afecta sobre todo al colon descendente. Un neumoperitoneo insuficiente y la existencia de adherencias que lo desplazan a la línea media favorecen su lesión. El olor fétido es el primer síntoma y la visualización de la luz intestinal es concluyente. Si el diagnóstico no es inmediato, la clínica se instaura a las pocos días con un cuadro de dolor abdominal y contractura de abdomen. Estaría indicada la laparotomía para llegar al diagnóstico, previa cobertura antibiótica y la colaboración de un cirujano digestivo. La gravedad del cuadro depende del grado de aseptización del intestino. La reparación o resección debe ir seguida de una ileostomía si la lesión afecta al colon ascendente, o de una colostomía en las demás localizaciones. El cierre debe hacerse dejando drenajes. El cierre de la colostomía o ileostomía se practica varios meses después.

\section{- Lesiones de intestino delgado}

Aunque las causas que provocan una lesión intestinal por el trócar son las mismas que hemos mencionado al referirnos al intestino grueso, debemos diferenciarlas porque su frecuencia es mayor $(0,16$ a $0,18 \%$ de las laparoscopias) y porque el tratamiento y la gravedad de las mismas son diferentes.

Las lesiones pueden ser sólo de la serosa, afectar parcialmente a la muscular, perforar la pared o provocar una perforación doble. El tratamiento puede ir, desde la observación clínica sin actuación inmediata, a la laparotomía para confirmar la extensión de las lesiones y su sutura si es posible. Si la perforación es amplia o si afecta a la irrigación sanguínea de la zona es mejor hacer una resección del segmento y anastomosis inmediata. Es aconsejable al menos la supervisión de un cirujano general. Si la lesión no se ha diagnosticado en el acto, o se ha optado por la observación y la paciente inicia un cuadro clínico de dolor, defensa abdominal y fiebre, la laparotomía debe ser inmediata y proceder en función de los hallazgos, que consistirá en resecar las zonas necróticas o no vascularizadas y recabar la presencia de un cirujano digestivo. No hay que olvidar que en estas circunstancias la peritonitis es grave y puede poner en peligro la vida de la enferma.

\section{Lesiones vesicales}

La punción vesical si pasa inadvertida puede provocar lesiones graves, sobre todo si la lesión que se produce es extraperitoneal.

Si se advierte, se debe realizar una sutura inmediata de la brecha y la colocación de una sonda permanente, como mínimo seis días, bajo protección antibiótica. Si la lesión pasa inadvertida y se observa en el post-operatorio (como oliguria o anuria, hematuria, ascitis...) una nueva exploración laparoscópica permitirá visualizar el orificio vesical en comunicación con el peritoneo y su sutura.

Si la lesión es extraperitoneal, la orina ocupa el espacio de Retzius y la oliguria es menor ya que el urinoma es de lenta evolución pero se palpan los labios mayores de la vulva edematosos e incluso puede llegar al muslo a nivel de la fascia. El riesgo es la fascitis necrotizante por E. Coli de evolución tórpida, grave y en ocasiones mortal.

\section{Hernias}

La aparición de hernia intestinal a través de los defectos de pared (trócares) son más frecuentes en los procedimientos que requieren múltiples entradas, extracción de grandes tumores, o uso de dilatadores que aumentan el daño de la fascia. La asociación Americana de Laparoscopistas Ginecológicos recogía 933 hernias sobre un total de 4.385 procedimientos laparoscópicos (incidencia del 21 por 100.000). El 80\% de estas hernias ocurrían a pesar del cierre de la fascia ${ }^{18}$. El riesgo es mayor en los sitios extraumbilicales y con el uso de trócares de $12 \mathrm{~mm}(3,1 \%)$ en relación con los de $10 \mathrm{~mm}(0,23 \%)$, por lo que la fascia debería ser cerrada en estas incisiones.

La manifestación clínica consiste en la presencia de un bulto, acompañado de dolor si están involucrados el intestino o el epiplon. Las náuseas y vómitos son signos de obstrucción intestinal. El tratamiento es el cierre secundario de la fascia (Tabla 1$)^{19}$. 
Tabla 1. Accidentes laparoscópicos por el trócar.

\begin{tabular}{lccc}
\hline \multicolumn{1}{c}{ Accidente } & $\mathbf{n}$ & $\begin{array}{c}\text { Intervenciones } \\
\mathbf{1 0 3 . 8 5 2} \text { (por mil) }\end{array}$ & $\begin{array}{c}\text { Punciones } \\
\mathbf{3 8 6 . 7 8 4} \text { (por mil) }\end{array}$ \\
\hline Hemorragia punto de punción & 57 & 2,10 & 0,60 \\
\hline Lesión vascular & 96 & 0,47 & 0,13 \\
\hline Lesión visceral & 14 & 0,63 & 0,16 \\
\hline Otros & 167 & 3,20 & 0,89 \\
\hline Total & 337 & & \\
\hline Mortalidad 7 casos (0,7 por mil) & &
\end{tabular}

\section{Lesiones durante la cirugía laparoscópica}

\section{Lesiones vasculares}

La frecuencia depende del tipo de cirugía endoscópica que se realice, de la alteración anatómica provocada por las lesiones previas y sobre todo de la habilidad del cirujano, pero oscila alrededor del $1 \%$. Las lesiones de las arterias y venas ilíacas pueden producirse excepcionalmente en las salpingectomías, anexectomías o histerectomías y requieren reparación inmediata.

Las lesiones vasculares por electrocirugía dan complicaciones post-operatorias cuando salta la escara y se produce la hemorragia.

Se remite su tratamiento a lo expuesto en lesiones vasculares secundarias a la introducción de trócares

\section{Lesiones intestinales}

Las lesiones intestinales durante la cirugía son poco frecuentes, pero debemos tener en cuenta que en la cirugía de la endometriosis, tanto ovárica como profunda, (endometriosis del tabique recto-vaginal) las adherencias del sigma y recto-sigma son frecuentes, gruesas y tan intimamente adheridas al tejido endometriósico que incluso la enfermedad puede perforar la víscera provocando rectorragias menstruales.

Para asegurarnos de que no se ha producido una perforación intestinal es necesario cubrir con suero Ringer la pelvis baja e insuflar aire por el ano clampando el sigma por encima de la zona disecada para excluir la lesión, por pequeña que sea, ésta dejará escapar unas burbujas de aire que borbotearán en el líquido.

La sutura será diferente si la lesión ocurre en el intestino grueso o delgado y depende del tamaño de la misma, el intestino delgado puede suturarse en dos capas y en el sigma la resección endoscópica debe decidirla y realizarla un cirujano digestivo.

Las lesiones a distancia o por contacto con un bisturí eléctrico por el calor de la pinza bipolar pueden dar clínica cuando salta la escara y la perforación intestinal se consuma. En estos casos, la mujer puede estar en su domicilio y el cuadro de peritonitis suele aparecer bruscamente, con lo que la gravedad aumenta y la defensa jurídica del cirujano es más difícil, que si la lesión se ha diagnosticado y tratado en el mismo acto quirúrgico o durante su estancia hospitalaria.

\section{Lesiones vesicales}

Durante la cirugía se puede producir lesión vesical en aquellos actos que implican su disección como la histerectomía total, la colposacrofijación, o la técnica Burch sobre todo si existe cirugía previa que distorsiona o elimina los planos de despegamiento.

Si la lesión es extraperitoneal y pasa inadvertida puede provocar fascitis necrotizante y septicemia fulminante.

\section{Lesiones ureterales}

Las lesiones ureterales incrementaron en frecuencia con la llegada del abordaje la- 
paroscópico. En la cirugía laparoscópica la magnificación disminuye este riesgo pero el principio básico es la identificación de su trayecto antes de proceder a cualquier cirugía en esta zona. Son especialmente expuestas la histerectomía total laparoscópica, la cirugia de la endometriosis y de la enfermedad inflamatoria pélvica. Además como factores predisponentes están los tumores pélvicos que pueden alterar la anatomía así como el antecedente de radioterapia. Los tres lugares más comunes son, en orden de frecuencia: a nivel de infundíbulo-pélvico, en el cruce a nivel de arteria uterina en el parametrio y en su entrada a la vejiga.

Puede producirse sección ureteral, lesión térmica, oclusión directa o estenosis por tracción, acodamiento o isquemia de un segmento ureteral.

La evolución clínica depende de la localización y del mecanismo lesional. Aproximadamente un 50\% de las obstrucciones ureterales unilaterales son asintomáticas. La lesión inadvertida puede manifestarse por un post-operatorio febril, con dolor lumbar que finalmente acaba produciendo una fistulización a vagina o a cavidad libre (ascitis urinosa). En este caso se apreciará una evolución post-operatoria tórpida, con frecuente íleo, distensión abdominal y cifras moderadamente elevadas de creatinina. Una oclusión ureteral inadvertida puede evolucionar a hidronefrosis con anulación de la función renal, con relativamente poca sintomatología, si no se acompaña de infección. En tales casos la lesión parenquimatosa renal puede, con el tiempo, convertirse en irreversible. La reparación de la lesión dependerá de su gravedad. En caso de sección limpia puede hacerse la anastomosis término terminal de primera intención, dejando un catéter para asegurar la permeabilidad del uréter. Si existe pérdida de algún segmento, será necesaria la reimplantación ureteral mediante la creación de una anastomosis con un trayecto submucoso antirreflujo a nivel de la cúpula vesical.

\section{Lesiones sobre masa anexiales de riesgo neoplásico}

La rotura capsular de un cáncer ovárico condiciona un cambio de estadio en la cla- sificación actual de la FIGO, tan importante como para indicar quimioterapia coadyuvante.

En caso de rotura capsular de una neoplasia ovárica, no se ha observado un cambio pronóstico siempre que la cirugía oncológica radical se suceda en un plazo inferior a 7-10 días o preferentemente en el mismo acto quirúrgico.

\section{Lesiones y accidentes en la extracción de la pieza quirúrgica}

- Accidentes intrínsecos a la naturaleza de la pieza

Ya hemos tratado la rotura de una cápsula neoplásica. En este mismo sentido, una extracción contaminante a través de la pared abdominal puede condicionar la aparición posterior de metástasis en los puntos de inserción. Deben manejarse con prudencia los contenidos de los quistes mucinosos y teratomas dermoides por su contendido irritante o adherencial. Si ocurre esta incidencia debe lavarse abundantemente la cavidad con suero caliente.

- Lesiones consecuentes a la vía de extracción

La imposibilidad de extracción de grandes piezas crean la necesidad de ampliación de las incisiones de los trócares dando lugar a la creación de hernias con o sin compromiso intestinal.

La culdotomía, incisión trasvaginal del saco de Douglas, resulta una excelente vía, pero debe conocerse suficientemente el método para evitar graves lesiones rectovaginales. Las maniobras de morcelación deben realizarse bajo un estricto control visual.

\section{COMPLICACIONES DE LA CIRUGÍA HISTEROSCÓPICA}

La histeroscopia es una técnica endoscópica para la visualización directa de la cavidad uterina y el canal endocervical mediante la introducción de una óptica y un medio de distensión. 
Se puede realizar con fines diagnósticos y/o terapéuticos (histeroscopia quirúrgica) con o sin anestesia (Fig. 6).

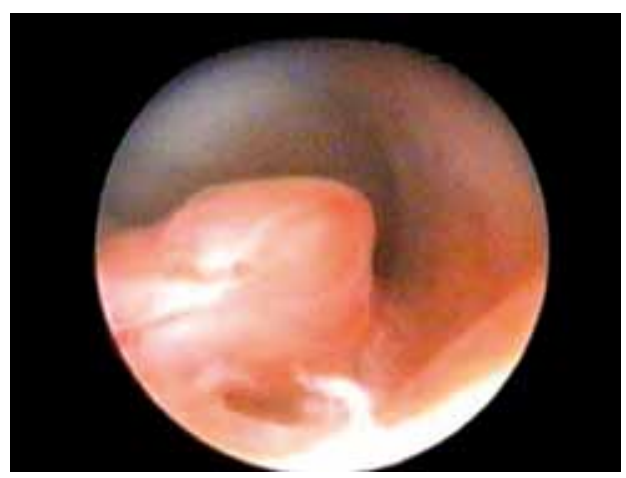

Figura 6. Pólipo endometrial.

\section{Derivadas de los medios de distensión}

\section{Distensión con $\mathrm{CO}$}

Embolia gaseosa Complicación muy rara y sólo posible si no se respetan los flujos y presiones.

\section{Medios de distensión líquidos}

- Solución salina/Ringer lactato los volúmenes usados en histeroscopia diagnóstica son inocuos. No se puede utilizar en procedimientos electroquirúrgicos.

- Glicina 1,5\% naúseas, vómitos, desorientación, con consecuencias tardías raras pero graves como amoniemia, encefalopatía y ceguera transitoria.

- Sorbitol/manitol naúseas, vómitos, desorientación.

Son complicaciones graves y cuando se produce requiere actuación conjunta con anestesiología, UCI y ginecología.

\section{Perforación uterina}

Es la más frecuente y su incidencia varía del 0,76 y $1,4 \%$. Puede aparecer tanto en el proceso de dilatación cervical como durante la utilización posterior del resector histeroscópico de forma mecánica o como injuria eléctrica. Ante su sospecha se debe interrumpir intervención, ingreso y antibióticos. Si se sospecha hemoperitoneo o lesión de órgano pélvico se debe acceder a la cavidad abdomino-pélvica. Cuando la perforación es electroquirúrgica, ante el riesgo de daño de órganos pélvicos siempre debe realizarse laparoscopia-laparotomía (Fig. 7).

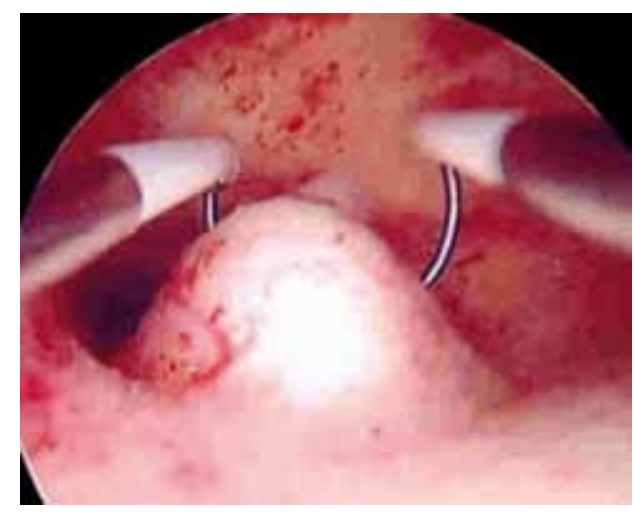

Figura 7. Resectoscopio.

\section{Complicaciones hemorrágicas}

De forma intraoperatoria se debe electrocoagular el lecho sangrante. Si no se cohíbe la hemorragia se debe introducir un catéter de Foley con 10-15 cc de suero fisiológico y retirarlo a las 3 horas. De forma post-operatoria son raras. La conducta a seguir es:

- Descartar lesiones cervicales

- Valorar la cuantía de pérdida sanguínea

- Sonda Foley intrauterina

- En casos extremos valorar histerectomía

\section{Infecciones}

La frecuencia es variable (menor del $2 \%$ y depende del proceso quirúrgico. Debe evitarse realizarlas en pacientes con infecciones agudas. La baja incidencia de esta complicación no justifica el uso sistemático de profilaxis antibiótica. Será el 
propio cirujano quien decida su uso de forma empírica en determinadas condiciones (intervenciones largas, pacientes con antecedentes de esterilidad-infertilidad).

\section{Hematometras}

Complicación tardía descrita en el 1-2\% de los casos de ablación o resección endometrial. Cursa con dolores crónicos y cíclicos en hipogastrio secundarios a la formación de un hematómetra por obstrucción del orificio cervical interno o adherencias intrauterinas. Se debe comprobar con ecografía y realizar permeabilización uterina ${ }^{20}$.

\section{BIBLIOGRAFÍA}

1. MagRina JF. Complications of laparoscopic surgery. Clin Obstet Gynecol 2002; 45: 469-480.

2. Wевв M, Symmonds R.Wertheim hysterectomy: a reappraisal. Obstet Gynecol 1979; 54; 140-145.

3. HARRIS WJ. Early complications of abdominal and vagynal hysterectomy.Obstet Gynecol Surv1995; 50: 795-780.

4. Sweet RL, GibBs RS. Antibiotic propgylaxis in obstetrics and gynecology, En: Sweet RL. Gibbs RS, eds. Infectious Diseases of the Female Genital Tract, $3^{\text {rd }}$ ed. Baltimore: Williams\&Wilkins, 1995: 729-745.

5. Mittendorf R, Aronson MP, Berry RE, Williams MA, Kupelnick B, KLickstein A et al. Avoiding serious infections associated with abdominal hysterectomy: A meta-analysis of antibiotic prophylaxis. Am J Obstet Gynecol 1993; 169: 1119-1124.

6. Wittmann DH, Schein M. Let us shorten antibiotic prophylaxis and therapy in surgery. Am J Surg 1996; 172 (suppl 6A): 26-32S.

7. Kingdom JCP, Kitchener GC, MacLean AB. Postoperative urinary tract infection in gynecology: Implications for an antibiotic prophylaxis policy. Obstet Gynecol 1990; 76: 636-638.

8. Finan MA, Barton DP, Fiorica JV, Hoffman MS, Roberts WS, GLEESON M et al. Ileus after gynecologic surgery: Management with water-so- luble hyperosmolar radiocontrast material. South Med J 1995; 88: 539-542.

9. Hartsell PA, Frazee RC, Harrison JB, SMith RW. Early postoperative feeding after elective colorectal surgery. Arch Surg 1997; 132: 518-520.

10. Dicker RC, Greenspan JR, Strauss LT, Cowart MR, Scally MJ, Peterson HB et al. Complications of abdominal and vaginal hysterectomy among women of reprodrctive age in the United States. The collaborative review of sterilization. Am J Obstet Gynecol 1982; 144: 841-848.

11. Montz FJ, Holschneider CH, Solh S, Schuricht LC, Monk BJ. Small bowel obstruction after radical hysterectomy: Risk factors, incidence, and operative findings. Gynecol Oncol 1994; 53: 114-120.

12. Vanrell JA, Carmona F. Complicaciones de cirugía ginecológica. En: Cañete ML, Cabero R. Urgencias en ginecología y obstetricia: Aproximación a la medicina basada en la evidencia. Madrid 2007: 611-639. Editorial FISCAM D.L.

13. Kuno L, Menzin A, Kauder H, Sison C, Gal D. Prophylactic ureteral catheterization in gynaecologic surgery. Urology 1998; 52: 1004-1008.

14. Hirsch HA, Kaser O, Ikle FA. Atlas de cirugía ginecológica. Quinta edición. Editorial Marban.

15. Mason A, Goldacre M, Meddings D, Woolfson J. Use of case fatality and readmission measures to compare hospital performance in gynaecology. B J Obstet Gynecol 2006; 113: 695.

16. Chapron C, Cravello L, Chopin N. Complications during set-up procedures for laparoscopy in gynecology: open laparoscopy does not reduce the risk, of major complications. Acta Obstet Gynecol Scand 2003: 82: 1125-1129.

17. Ansen FW, KolkmanW, Bakkum EA, De Kroon CD, Trimbos-Kemper TC, Trimbos JB. Complications of laparoscopy: an inquiry about closedversus open-entry technique. Am J Obstet Gynecol 2004; 190: -334-634.

18. Montz FJ, Holschneider CH, Munro MG. Incisional hernia following laparoscopy: a survey of the American Association of Gynecologic Laparoscopists. Obstet Gynecol 1994; 84: 881.

19. Champault G, Cazaru G. J Chir (Paris) 1995; 132: 109-113.

20. S.E.G.O. Histeroscopia Documento de Consenso 1996: 1-47 (www.SEGO.es) 
